\title{
A novel colon cancer gene therapy using rAAV-mediated expression of human ShRNA-FHL2
}

\author{
YAO WU $^{1 *}$, ZHENG GUO $^{2 *}$, DI ZHANG ${ }^{1}$, WENJING ZHANG ${ }^{1}$, QINGQING YAN $^{1}$, XINPENG SHI $^{1}$, \\ MENGNAN ZHANG $^{3}$, YINGYING ZHAO ${ }^{1}$, YALI ZHANG ${ }^{1}$, BO JIANG ${ }^{1}$, \\ TIANMING CHENG $^{1}$, YANG BAI $^{1}$ and JIDE WANG ${ }^{1}$ \\ ${ }^{1}$ Guangdong Provincial Key Laboratory of Gastroenterology, Department of Digestive Medicine, \\ Nanfang Hospital, Southern Medical University, Guangzhou 510515; ${ }^{2}$ Department of Oncology, \\ The 1st Affiliated Hospital of Gannan Medical University, Ganzhou 341000; ${ }^{3}$ Department of Gastroenterology, \\ The 1st Affiliated Hospital of Guangzhou Medical University, Guangzhou 510210, P.R. China
}

Received June 24, 2013; Accepted August 5, 2013

DOI: 10.3892/ijo.2013.2090

\begin{abstract}
FHL2 (Four and a half LIM-only protein 2) has been identified as an oncogene in colon cancer and suppression of FHL2 induces cell differentiation and tumorigenesis in colon cancer cell lines. The aim of this study was to develop a novel and effective approach to knockdown FHL2, which can serve as a promising target of colon cancer therapy. Recombinant adeno-associated virus (rAAV) was generated bearing with FHL2-shRNA and transfected into LoVo cells. Cell cycle and growth were assessed. The interaction between FHL2 and G0/G1 cell cycle and growth was evaluated by flow cytometry, western blot analysis and WST-1 assay. We showed that suppression of FHL2 by rAAV-shRNA induced G0/G1 cell cycle arrest and inhibited cell growth. Apoptosis-related proteins and their activity was investigated at the same time. rAAV-FHL2-shRNA activated intrinsic and extrinsic apoptotic pathways and increased cell susceptibility to apoptotic stimuli by 5 -FU. Moreover, a xenograft model was established to explore rAAV-FHL2-shRNA with 5-FU mediated tumorigenesis in vivo. A strong anti-tumorigenic effect of rAAV-FHL2-shRNA was shown in nude mice and this antitumor effect was enhanced when combined with 5-FU
\end{abstract}

Correspondence to: Dr Jide Wang or Dr Yang Bai, Department of Digestive Medicine, Nanfang Hospital, Southern Medical University, 1838 North Guangzhou Avenue, Guangzhou 510515, P.R. China

E-mail: jidewang55@163.com

E-mail: baiyang1030@hotmail.com

*Contributed equally

Abbreviations: FHL2, four and a half LIM-only protein 2; AAV, adeno-associated virus; EGFP, enhanced green fluorescence protein; PI, propidium iodide

Key words: four and a half LIM-only protein 2, adeno-associated virus, gene therapy, cell cycle, apoptosis, colon cancer treatment. These findings implicate FHL2 as a cell cycle and growth modulator and thus inhibit apoptosis in colon cancer cells. rAAV-shRNA-FHL2 may serve as a novel and potent therapeutic or 5-FU co-therapeutic agent for colon cancer.

\section{Introduction}

FHL2 is a LIM-only protein that belongs to the four and a half LIM-only protein family. It consists of four LIM domains and one N-terminal half LIM domain. The abbreviation LIM represents the names of three transcription factors (Lin-11, Isl-1 and Mec-3) in which such a domain was first identified (4). It is strongly expressed in cardiac and skeletal muscle cells but a much lower level was observed in other tissues and cell types (1-4). The LIM domains are double zinc finger motifs that play multiple roles in protein-protein interaction (5). FHL2 can interact with over 50 different proteins with diverse functions such as receptors, enzymes, transcription factors, cofactors or splicing factors (6). Thus, FHL2 is involved in the regulation of various cellular processes including proliferation, differentiation, migration, adhesion, motility and contraction.

The role of FHL 2 in cancer is particularly intriguing since FHL2 binds to different proteins and can function in a cell-type dependent fashion as transcriptional co-activators of several transcription factors, including androgen receptor, AP-1, CREB, BRCA1, WT-1 and NF- $\kappa$ B in various transformed cell types (7-11), or as transcriptional co-repressors of ERK2, PLZF, SRF and FOXO1 (12-15). FHL2 was first identified as being downregulated in human rhabdomyosarcoma cells, suggesting a suppressor role in tumor development (16). Many recent studies have reported the differential expression of FHL2 in tumor tissues. Interestingly, FHL2 is overexpressed in breast cancer (17), prostate cancer (18), ovarian cancer (4), gastrointestinal cancer (19) and glioma (20) but downregulated in liver cancer (21), making the role of FHL2 in cancer development elusive. It is also notable that FHL2 triggers apoptosis in human RD, monkey kidney COS-1 and normal mouse fibroblast NIH 3T3 cell lines (22) but plays an anti-apoptotic role in glioblastoma (20). The intriguing aspects of FHL2 
being as oncoprotein or tumor suppressor may be related to its interaction with different partner proteins even in different cell types (23). Suppression of FHL2 was detected to induce cell differentiation and inhibit tumorigenesis in FHL2 high expression colon cancer cells, while in HT29 cells with bare FHL2 expression, attenuated FHL2 suppressed cell growth and differentiation $(19,23)$. We and other groups have reported that FHL2 was also a potent EMT inducer by stimulating vimentin and MMP-9 expressions and causing a loss of E-cadherin in colon DLD1 cells (24) and FHL2 negatively regulated the transcription of E-cadherin through interaction with Snaill (25). For the tissue and cellular function specificity, FHL2 might be the target in cancer biological therapy.

In our study, we have demonstrated that a novel and effective way to knockdown FHL2, the rAAV-FHL2-shRNA can induce apoptosis significantly, inhibit tumorigenesis and tumor growth, strongly enhance the antitumor activity of 5-FU and markedly prolonged the survival time of animals bearing tumor xenografts in vivo. Our results document that rAAV-shRNA-FHL2 is a promising candidate for gene therapy of colon cancer.

\section{Materials and methods}

Cell culture. Culture reagents were purchased from Invitrogen (Carlsbad, CA, USA). Colon cancer cell line LoVo was obtained from American Type Culture Collection (Rockville, MD, USA) and cultured as described (19). Cell was maintained at $37^{\circ} \mathrm{C}$ in a $5 \% \mathrm{CO}_{2}$ humidified incubator and subcultured using $0.25 \%$ trypsin every 2-3 days before confluence was reached.

Constructs. As we have described previously (20), the enhanced green fluorescence protein (EGFP) was constructed by inserting the EGFP gene between the XhoI and EcoRI sites of the AAV2 vector. Short hairpin RNA (shRNA) targeting FHL2 (sense: 5'-TCGACGCGAATCTCTCTTTGGCAAGTTCAAGAG ACTTGCCAAAGAGAGATTCGTTTTTTGGAAT-3'; antisense: 5'-CTAGATTCCAAAAAACGAATCTCTCTTTGG CAAGTCTCTTGAACTTGCCAAAGAGAGATTCGCG-3') or luciferase (sense: 5'-TCGACGCGTACGCGGAATACT TCGATTCAAGAGATCGAAGTATTCCGCGTACGTTTT TTGGAAT-3'; antisense: 5'-CTAGATTCCAAAAAACGT ACGCGGAATACTTCGATCTCTTGAATCGAAGTATTC CGCGTACGCG-3') was initially inserted into the SalI and $X b a \mathrm{I}$ sites of pAVU6+7 plasmid and then the construct was sub-cloned into the AAV2 expression plasmid in order to substitute the CMV promoter with the U6 promoter.

Western blot analysis. The whole cell lysates were prepared with lysis buffer $(20 \mathrm{mM}$ Tris- $\mathrm{HCl}, 1 \mathrm{mM}$ EDTA, $1 \mathrm{mM}$ EGTA, $1 \mathrm{mM}$ sodium vanadate, $0.2 \mathrm{mM}$ phenylmethylsulfonyl fluoride, $0.5 \%$ NP-40, $1 \mu \mathrm{g} / \mathrm{ml}$ leupeptin, $1 \mu \mathrm{g} / \mathrm{ml}$ aprotinin, and $1 \mu \mathrm{g} / \mathrm{ml}$ pepstatin A). In total, 10 or $30 \mu \mathrm{g}$ of cell lysate was subjected to SDS-PAGE, transferred to PVDF membranes, and probed with first antibodies against CDK6, cyclin D, procaspase 3 , cleaved caspase 3 , procaspase 8 , cleaved caspase 8 (Santa Cruz Biotechnology, Santa Cruz, CA, USA), procaspase 9 (Alexis Biochemical, San Diego, CA, USA), cleaved caspase 9 (Imgenex, San Diego, CA, USA) or FHL2 (MBL International Incorporation, Woburn, Japan) followed by the HRP-conjugated secondary antibody. Goat antihuman actin antibody (I-19, Santa Cruz Biotechnology) was used as internal control. Antigen-antibody complexes were visualized by the enhanced chemiluminescence (ECL) system (Amersham Biosciences, Little Chalfont, UK).

Preparation of $r A A V$. The rAAV particles were produced using a helper virus free system as previously described in HEK 293 cells (20). The viruses were purified by HiTrap Heparin column chromatography (Sigma Chemical Co., St. Louis, MO, USA) and viral titer was determined by real-time PCR using the SYBR-Green I kit (Applied Biosystems, Foster City, CA, USA) with a forward primer (5'-CGGCTGTTGGGCACTGA-3') and a reverse primer (5'-CCGAAGGGACGAAGCAGAAG-3'). Aliquot of viral stocks $\left(1.5 \times 10^{12}\right.$ viral genomes $\left./ \mathrm{ml}\right)$ were stored at $-80^{\circ} \mathrm{C}$ before use.

Gene transfection in vitro. LoVo cells were cultured in 6-well plastic plates and transfected with rAAV-EGFP or rAAV-FHL2-shRNA at different multiplicity of infection $\left(\mathrm{MOI}=1 \times 10^{4}, 1 \times 10^{5}, 5 \times 10^{5}\right)$. From then on, the enhanced green fluorescent protein (EGFP) was observed under a fluorescent microscope and the expression of rAAV-FHL2-shRNA was detected by western blot analysis.

Flow cytometry scan. To analyze cell cycle, cells were collected and fixed with ice-cold $70 \%$ ethanol in PBS and stored at $-4^{\circ} \mathrm{C}$ until use. After resuspension, cells were incubated with $100 \mu \mathrm{l}$ of RNase $\mathrm{I}(1 \mu \mathrm{g} / \mathrm{ml})$ and $100 \mu \mathrm{l}$ of propidium iodide (PI) $(400 \mu \mathrm{g} / \mathrm{ml})$ at $37^{\circ} \mathrm{C}$ and analyzed by flow cytometry (Becton-Dickinson, Franklin Lakes, NJ, USA). The cell cycle phase distribution was calculated from the resultant DNA histogram using Multicycle AV software (Phoenix Flow Systems, San Diego, CA, USA). Apoptosis was detected using the Annexin V-FITC kit according to the manufacturer's instructions (Trevigen Inc., Gaithersburg, MD, USA). Briefly, cells with various treatments were collected and stained with Annexin V-FITC and PI in the dark for $15 \mathrm{~min}$ at room temperature. After addition of binding buffer, cells were analyzed by flow cytometry and analyzed using Winmdi 2.8 .

Cell viability assay. The colorimetric WST-1 assay was performed to assess the effect of rAAV-FHL2-shRNA on cell proliferation (26). The measurement was based on the ability of viable cells to cleave the sulfonated tetrazolium salt WST-1 (4-(3-(4-iodophenyl)-2-(4-nitrophenyl)2H-5-tetrazolio)-1,3-benzene disulfonate) by mitochondrial dehydrogenases. LoVo cells (5,000 cells/well) were plated in a 96-well plate in regular growth medium, and after $16 \mathrm{~h}$ the medium was replaced with $2 \%$ FBS containing medium. After $72 \mathrm{~h}, 10 \mu \mathrm{l}$ WST-1 reagent was added in each well followed by additional incubation for $2 \mathrm{~h}$. The absorbance at $450 \mathrm{~nm}$ was measured using a microplate reader.

Morphological detection of apoptosis. Morphological evaluation of apoptotic cell death was performed as previously described with some modification (27). Cells were fixed for $5 \mathrm{~min}$ in $3 \%$ paraformaldehyde in phosphate-buffered saline (PBS). After air-drying, cells were stained for $10 \mathrm{~min}$ in Hoechst $33258(10 \mu \mathrm{g} / \mathrm{ml})$, mounted in $50 \%$ glycerol 
containing $20 \mathrm{mM}$ citric acid and $50 \mathrm{mM}$ orthophosphate, and stored at $-20^{\circ} \mathrm{C}$ before analysis, nuclear morphology was evaluated using a Zeiss IM 35 fluorescent.

Caspase 3, 8, 9 activity assay. Caspase 3, 8, 9 activity was determined using the ApoAlert caspase colorimetric assay kit according to the manufacturer's instructions (Clontech, Moutain View, CA, USA) (28). Briefly, assays were performed on 96-well microtiter plates by incubating $10 \mu \mathrm{l}$ protein of cell lysate per sample in $80 \mu \mathrm{l}$ reaction buffer [1\% NP-40, $20 \mathrm{mmol} / \mathrm{l} \mathrm{Tris}-\mathrm{HCl}$ (pH 7.5), $137 \mathrm{mmol} / 1 \mathrm{Nad}$, and $10 \%$ glycerol] containing $10 \mu \mathrm{l}$ caspase 3, 8,9 substrate (Ac-DEVDpNA) ( $2 \mathrm{mmol} / \mathrm{l})$. Lysates were incubated at $37^{\circ} \mathrm{C}$ for $4 \mathrm{~h}$. Samples were measured with an enzyme-linked immunosorbent assay reader at an absorbance of $405 \mathrm{~nm}$. All of experiments were performed at least 4 times.

Experimental animal model and tumorigenesis assay. Five to six-weeks-old female BALB/c nude mice were bred under pathogen-free conditions at the Southern Medical University (Guangzhou, China). All animal studies were approved by the Southern Medical University Animal Care and Use Committee. LoVo cells in exponential growth phase were harvested and washed twice in PBS. The cells were resuspended in PBS at a density of $5 \times 10^{7}$ cells $/ \mathrm{ml}$ and $0.1 \mathrm{ml}\left(5 \times 10^{6}\right.$ cells) of the cell suspension was then injected subcutaneously into the right flank of each nude mouse (29). Seven days after subcutaneous tumor cell injection, mice were anesthetized and $1.5 \times 10^{11} \mathrm{v}$.g. of rAAV-Luc-shRNA, rAAV-FHL2-shRNA, rAAV-Luc-shRNA + 5-FU or rAAV-FHL2-shRNA + 5-FU was injected directly into the tumors at three different locations by a 10 -ml micro-syringe (Hamilton, Reno, NV, USA). Four mice (4 injections) were included in each group (each transduced cell line). The volumes of tumor were calculated as follows: $V=(4 / 3) R 1^{2} R 2$, where $R 1$ is radius 1 and $R 2$ is radius 2 and $\mathrm{R} 1<\mathrm{R} 2$. Mice were sacrificed and tumors were dissected and weighed on day 35 after inoculation.

Statistical analysis. Data are presented as the mean \pm standard error of mean (SEM). The significance of the difference between groups was evaluated with the Student's t-test or one-way ANOVA test. $\mathrm{P}<0.05$ was considered significant difference.

\section{Results}

Concentration and time kinetics of rAAV transduction in LoVo cell. LoVo cells were infected with rAAV-EGFP at different multiplicity of infection (MOIs) ranging from $1 \times 10^{4}$ to $5 \times 10^{5}$. Forty-eight hours after infection, expression of EGFP was analyzed for by flow cytometry (30). At a MOI of $1 \times 10^{4}$, an average of $13.6 \%$ of cells $(\mathrm{SEM}=0.2 \%)$ was EGFP positive. At a MOI of $1 \times 10^{5}$, a significant increase of positive cells up to $40.1 \%$ (SEM=1.2\%) was observed. At a MOI of $5 \times 10^{5}$, a slight but insignificant increase of EGPF cells was detected $53.7 \%$; SEM=4.0\%; Fig. 1A, one-way ANOVA analysis). As shown in Fig. 1B, FHL2 expressed at high level in LoVo cells, while with different dosage rAAVFHL2-shRNA transduction, FHL2 expression decreased dose-dependently.

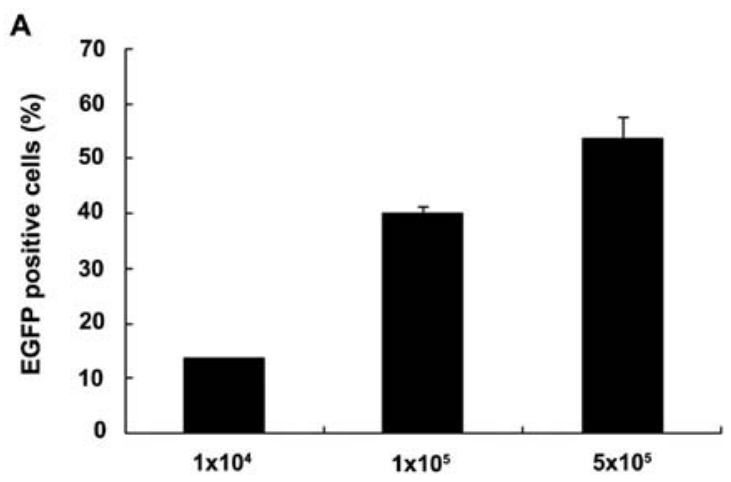

B

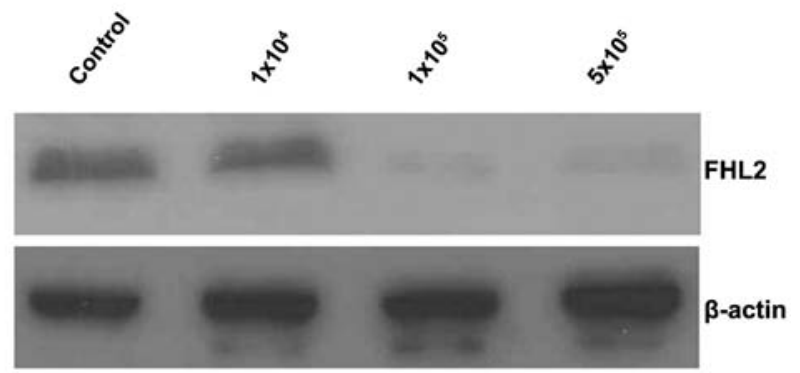

Figure 1. Concentration kinetics of rAAV-EGFP and rAAV-FHL2-shRNA. (A) LoVo was infected with rAAV-EGFP at a MOI of $0,1 \times 10^{4}, 1 \times 10^{5}$ or $5 \times 10^{5}$. Flow cytometry was performed to analyze expression of EGFP $48 \mathrm{~h}$ after infection. (B) LoVo was infected with rAAV-FHL2-shRNA at a MOI of 0 , $1 \times 10^{4}, 1 \times 10^{5}$ or $5 \times 10^{5}$, whole lysate was collected and FHL2 expression was detected by western blot analysis. $\beta$-actin was used as the internal control.

With this efficient gene interference by rAAV, FHL2 was specifically and highly inhibited. No significance decrease in $5 \times 10^{5}$ titer, suggested $1 \times 10^{5}$ as the most appropriate MOI in the future experiments.

rAAV-FHL2-shRNA induces G0/G1 cell cycle arrest. To further address effect of FHL2 on the cell cycle, we employed rAAV vectors expressing either FHL2-shRNA or Luc-shRNA to infect LoVo cells at a dose of $1 \times 10^{5}$ MOI. Flow cytometry revealed that rAAV-FHL2-shRNA transduction led to significant G0/G1 phase accumulation in LoVo cells (Fig. 2A).

The cyclin-dependent kinases together with the cyclin D proteins are specifically involved in the progression of cells through the G1 phase and the entry into the S phase. Here, the protein expression of cyclin D1 and CDK6 was analyzed in total protein lysates after transduced with FHL2-shRNA. Cyclin D1 and CDK6 were decreased in rAAV-FHL2-shRNA compared with rAAV-Luc-shRNA (Fig. 2B).

Ectopic expression of FHL2-shRNA suppresses cell growth. WST-1 assay is commonly used to estimate cell survival, growth, and differentiation (26). The cells with rAAV-Luc-shRNA was considered as control, cultured in $0.4,2.0$ and $10 \%$ FBS for $72 \mathrm{~h}$. The OD 450 values of control group were $0.672 \pm 0.031,1.284 \pm 0.162$ and $2.236 \pm 0.091$; whereas those of rAAV-FHL2-shRNA were $0.482 \pm 0.083$, $0.662 \pm 0.028$ and $0.826 \pm 0.209$, respectively (Fig. 2 C). Ectopic expression of rAAV-FHL2-shRNA suppressed cell growth in a serum-dependent manner $(\mathrm{P}<0.05)$. 
A

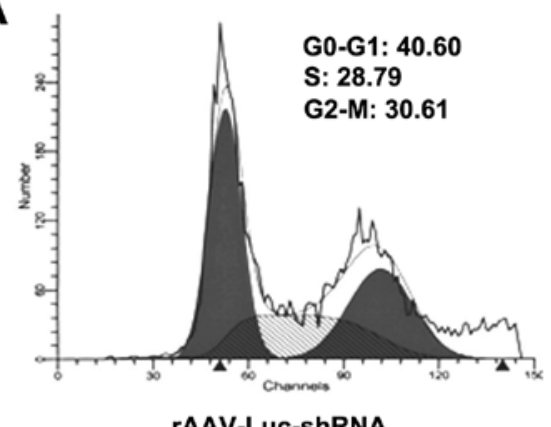

rAAV-Luc-shRNA

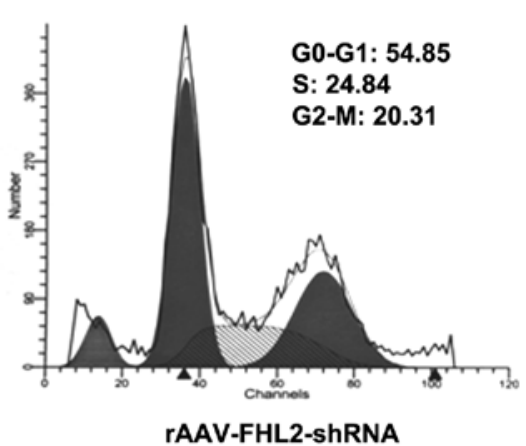

rAAV-FHL2-ShRNA

B

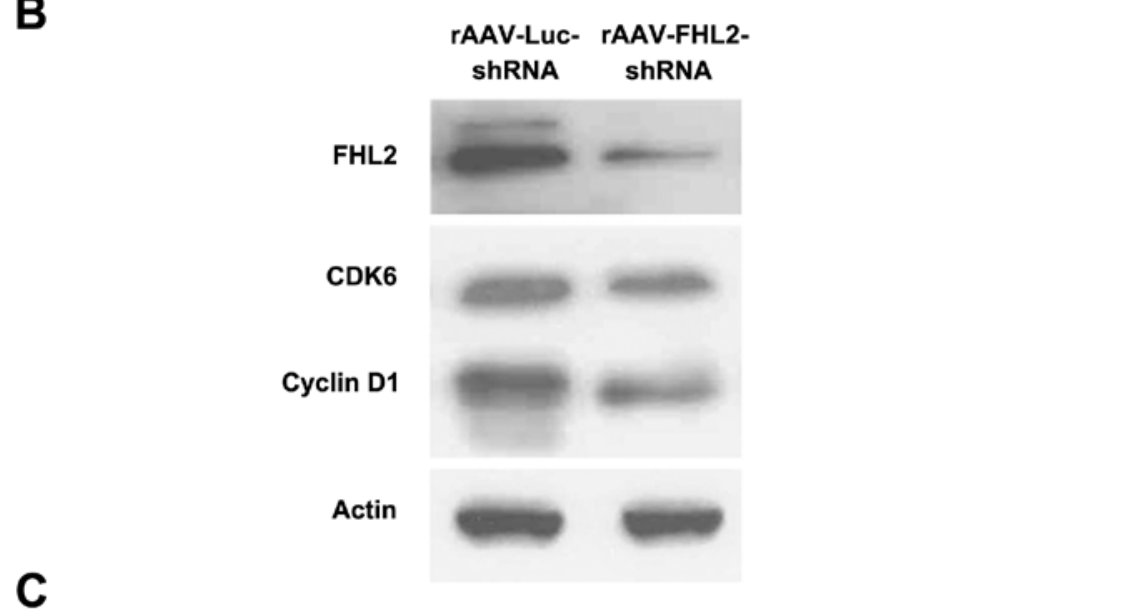

C

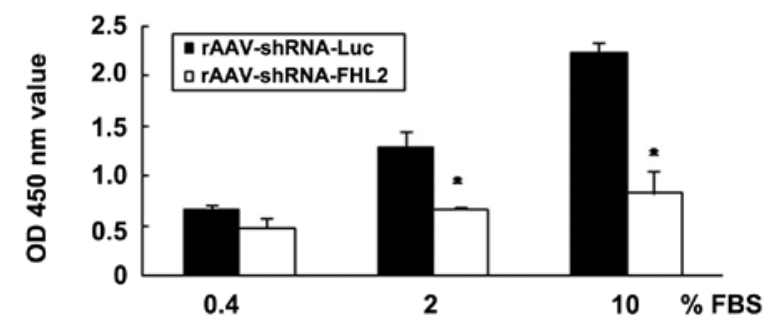

D

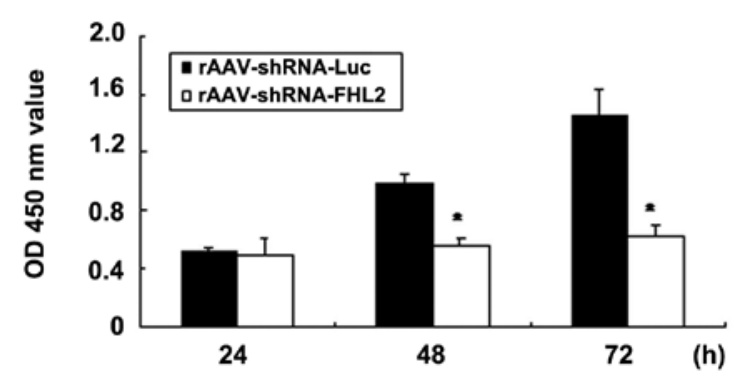

Figure 2. The effect of rAAV mediated FHL2-shRNA expression on cell cycle progression and cell growth. (A) rAAV-Luc-shRNA or rAAV-FHL2-shRNA (MOI $1 \times 10^{5}$ ) was transduced into LoVo cells. Cell populations were analyzed by FACS at $48 \mathrm{~h}$ post-transduction. (B) The expression of cell cycle related proteins was detected in LoVo cells infected with rAAV-Luc-shRNA or rAAV-FHL2-shRNA at a MOI 1x10 5 for $48 \mathrm{~h}$. (C) LoVo cells were seeded in 96-well tissue culture plates and were exposed with $\mathrm{r} A A V$-Luc-shRNA or $\mathrm{r} A A V$-FHL2-shRNA. Cell proliferation was assessed by WST-1 assay after 72-h culture with different serum concentration ("P $<0.05$, comparing to $\mathrm{r} A A V$-Luc-shRNA). (D) LoVo cells with $\mathrm{r} A A V$-Luc-shRNA or rAAV-FHL2-shRNA were cultured in complete medium for 24,48 and $72 \mathrm{~h}$, proliferation was evaluated. The absorbance values at $450 \mathrm{~nm}$ are expressed as means $\pm \operatorname{SEM}\left(\mathrm{n}=3 ;{ }^{*} \mathrm{P}<0.05\right)$.

We assessed the proliferation of rAAV-FHL2-shRNA cells cultured in complete medium (5\% FBS) for various time points. The OD 450 of rAAV-Luc-shRNA were $0.522 \pm 0.022$, $0.986 \pm 0.062$ and $1.456 \pm 0.152 \%$ with 24,48 and $72 \mathrm{~h}$ culture individually, whereas those of rAAV-FHL2-shRNA cells were inhibited as $0.496 \pm 0.124,0.562 \pm 0.041$ and $0.62 \pm 0.084 \%$, respectively (Fig. 2D, P $<0.05$ ).
rAAV-FHL2-shRNA activates intrinsic and extrinsic apoptotic pathways. To investigate the mechanism of rAAV-FHL2shRNA induced growth suppression, apoptosis was assayed by flow cytometry. As shown in Fig. 3A, rAAV-FHL2-shRNA induced more apoptosis than rAAV-Luc-shRNA in LoVo cells, indicating that rAAV-FHL2-shRNA inhibits cell growth by inducing apoptosis. 
A

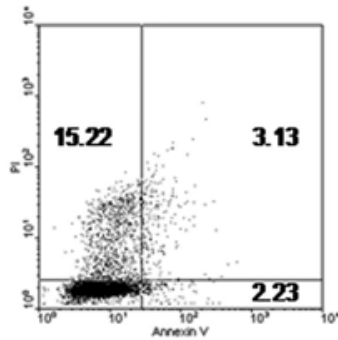

PI

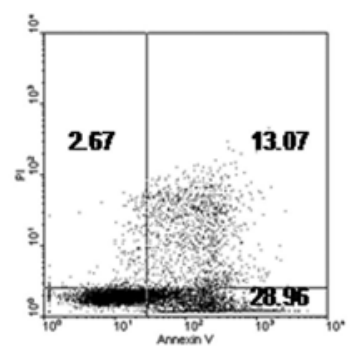

rAAV-ShRNA-FHL2

Annexin V
B

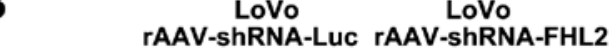

rAAV-ShRNA-Luc rAAV-ShRNA-FHL2

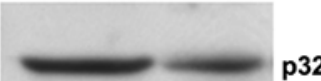

Cleaved caspase 3

Procaspase 8

Cleaved caspase 8

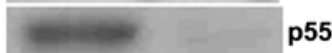

caspase 8

Procaspase 9

Cleaved caspase 9

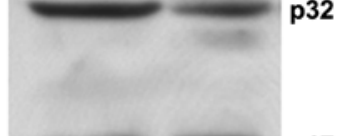

Cleaved caspase 9

Actin

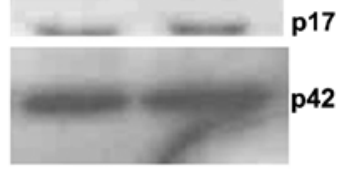

$\mathbf{C}$

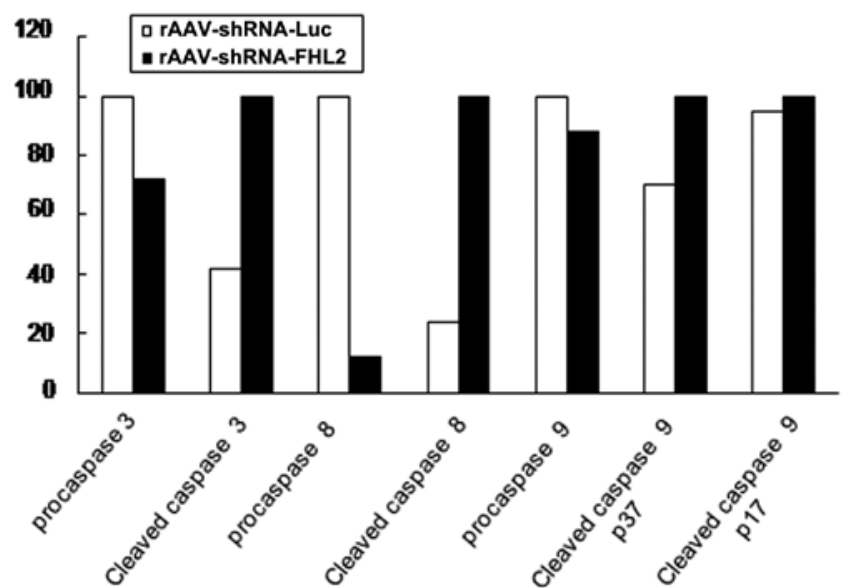

D

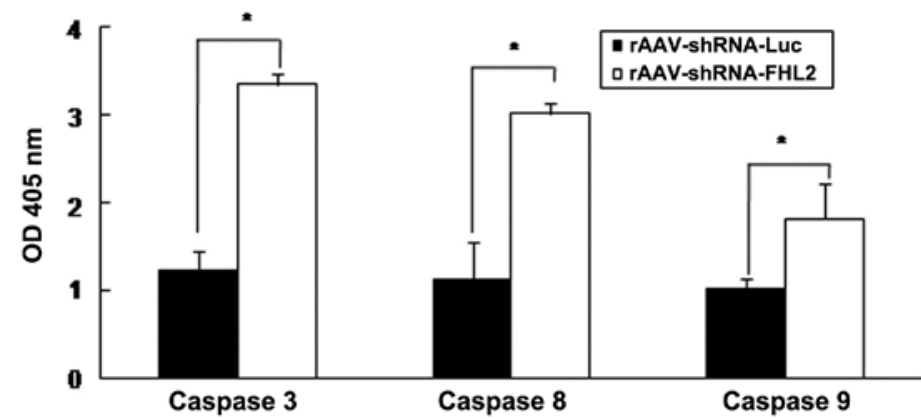

Figure 3. Suppression of rAAV-FHL2-shRNA activated intrinsic and extrinsic apoptotic pathways. (A) r $A A V$-Luc-shRNA and rAAV-FHL2-shRNA were transduced. Apoptosis was detected by FACS analysis $48 \mathrm{~h}$ later with PI and Annexin V-FITC double staining. All of these experiments were repeated two to three times. (B and C) LoVo cell lysates were prepared $48 \mathrm{~h}$ post-transduction. The expression of pro- and cleaved caspases 3,8 and 9 were detected by western blot analysis with $\beta$-actin as the internal control. (C) The density of the bands relative to that of $\beta$-actin band was calculated. (D) The activity of caspases 3,8 and 9 were detected $48 \mathrm{~h}$ after transduction. The value of OD $405 \mathrm{~nm}$ was calculated individually in each group ( $\mathrm{P}<0.05$, comparing to rAAV-Luc-shRNA).

Caspases are essential in cells for apoptosis and have been termed 'executioner' proteins for their roles (28,31-33). rAAV-FHL2-shRNA activated caspases 3, 8 and 9 as evident by the increasing protein level of cleaved caspases (Fig. 3B and C). Moreover, the activity of caspases 3,8 and 9 was analyzed after rAAV-shRNA-FHL2 transduction. The ratio between OD $405 \mathrm{~nm}$ of transfected cells and OD $405 \mathrm{~nm}$ of parental cells was calculated. The activity of caspases 3,8 and 9 was significantly increased in rAAV-FHL2-shRNA compared with rAAV-Luc-shRNA (Fig. 3D).

Suppression of rAAV-FHL2-shRNA increases cell susceptibility to apoptotic stimuli. To evaluate the role of rAAV-FHL2-shRNA in chemotherapy-induced apoptosis, the 
A
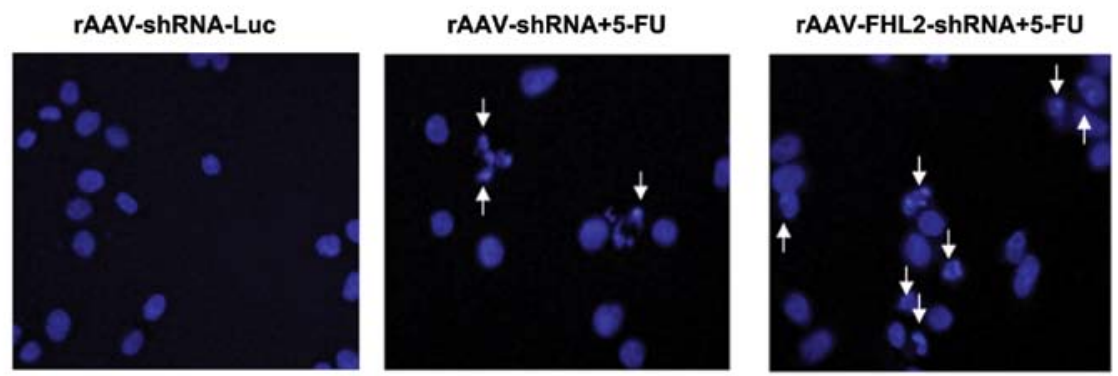

\section{B}

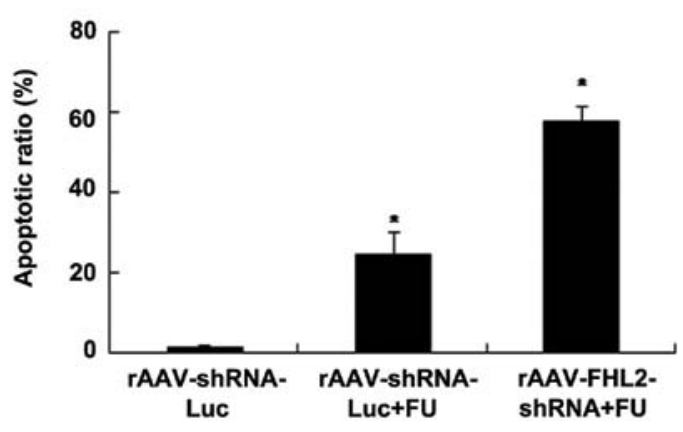

C
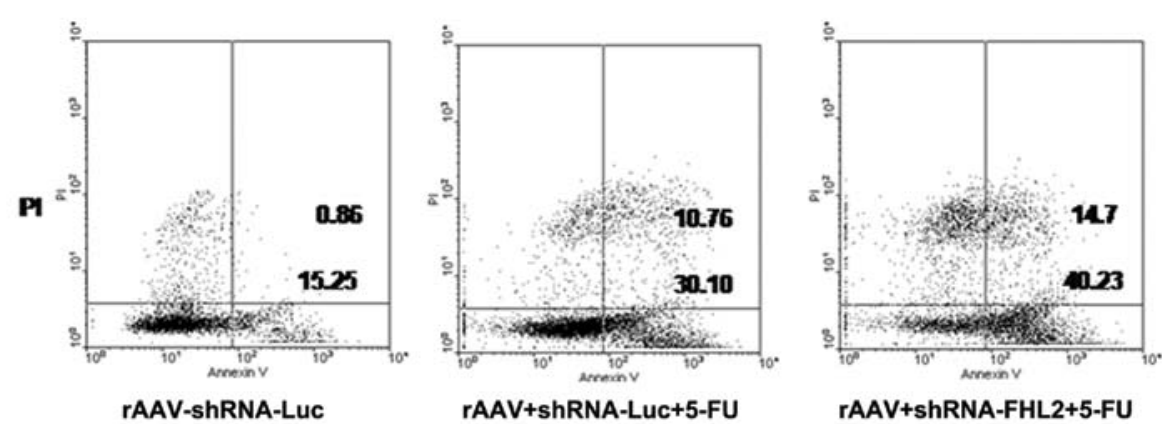

rAAV+shRNA-Luc+5-FU

rAAV+shRNA-FHL2+5-FU

Annexin V

Figure 4. Suppression of rAAV-FHL2-shRNA increased cell susceptibility to apoptotic stimuli. (A) LoVo cells were treated with rAAV-Luc-shRNA, rAAV-Luc-shRNA + 5-FU or rAAV-FHL2-shRNA + 5-FU. Nuclei were stained with Hoechst 33258 and visualized under a fluorescent microscope (arrow represented cell with nuclear fragmentation and condensed chromatin). (B) Illustration of apoptotic cells after staining with Hoechst 33258. The values are expressed as the means \pm SEM from 3 separate experiments. "P<0.05 between the 2 transfectants. (C) Cells with rAAV-Luc-shRNA or rAAV-FHL2-shRNA transduction were treated with 5-FU for $48 \mathrm{~h}$, double stained with Annexin V-FITC and PI, followed by flow cytometry analysis to determine apoptosis.

cells were transduced with rAAV, treated or not with 5 -FU (50 $\mu \mathrm{g} / \mathrm{ml}$ in NS) (34), apoptotic morphological changes was analyzed by staining with Hoechst 33258 blue fluorescence; a brightly blue-fluorescent condensed nuclei and chromatin fragmentation was considered as apoptosis by fluorescence microscopy (Fig. 4A). Similarly, the ratios of condensed nuclei positive cells were higher in rAAV-FHL2-shRNA + 5-FU of LoVo cells comparing with the rAAV-Luc-shRNA and rAAV-Luc-shRNA + 5-FU controls $(\mathrm{P}<0.05$ comparing with rAAV-Luc-shRNA control) (Fig. 4B). Moreover, rAAV-Luc-shRNA and rAAV-FHL2-shRNA cells were treated with 5-FU for $48 \mathrm{~h}$ (NS was used as vehicle), double stained with Annexin V-FITC and PI, followed by flow cytometry analysis to determine the apoptosis. As shown in Fig. 4C, the apoptotic index of rAAV-Luc-shRNA + 5-FU and rAAV-FHL2-shRNA + 5-FU was significantly increased relative to rAAV-Luc-shRNA controls.
These findings suggested that rAAV-FHL2-shRNA enhanced the susceptibility of cancer cells to apoptotic triggers induced by $5-\mathrm{FU}$.

In vitro transduction of rAAV-FHL2-shRNA inhibits tumor formation in vivo. Moreover, the antitumor effect of rAAV-FHL2-shRNA and/or 5-FU in vivo was demonstrated by a xenograft model in nude mice. LoVo cells were subcutaneously injected in the right flanks of the nude mice. When the tumor nodules became visible (about 3-5 $\mathrm{mm}$ in diameter), rAAV-Luc-shRNA or rAAV-FHL2-shRNA was injected directly into the tumor and 5-FU was intraperitoneally injected as a co-treatment. The tumor sizes were continuously monitored on a weekly basis. As shown in Fig. 5, the tumor volumes of the rAAV-FHL2-shRNA and rAAV-FHL2-shRNA + 5-FU treated mice were markedly smaller than those of the rAAV-Luc-shRNA treated mice 4 weeks after rAAV injection. 
A

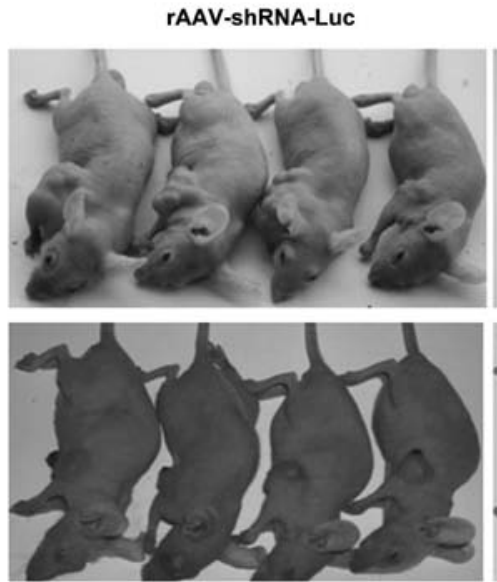

rAAV-ShRNA-LUC+5-FU

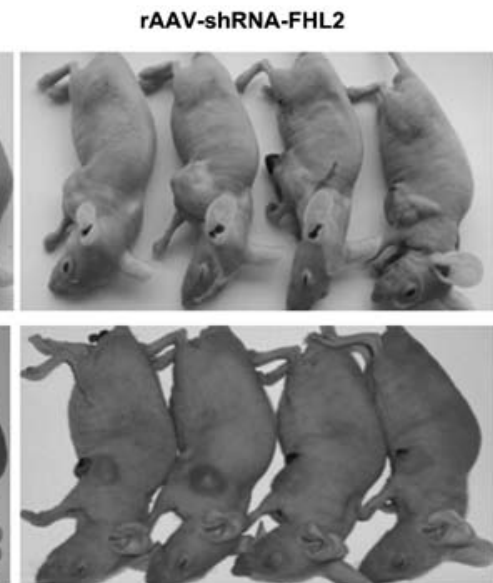

rAAV-ShRNA-FHL2+5-FU

B

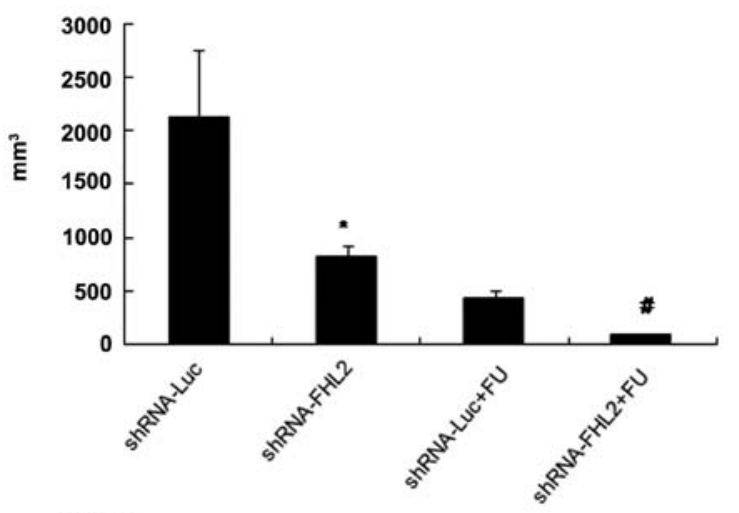

C

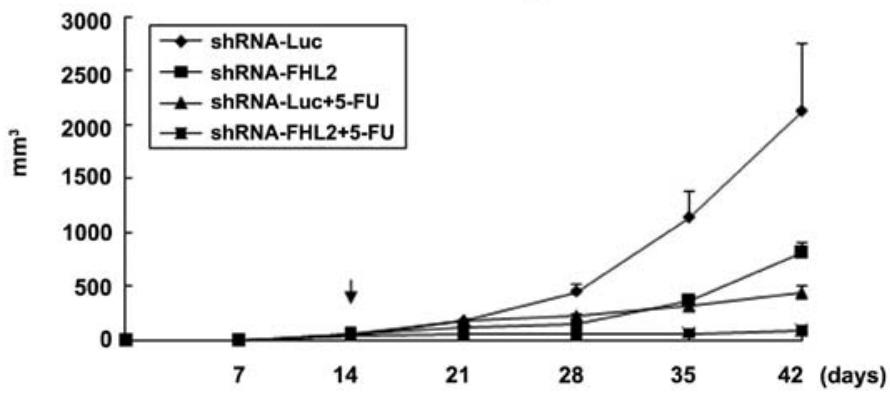

Figure 5. In vivo transduction of rAAV-shRNA-FHL2 combined with 5-FU treatment significantly inhibited tumor formation. (A) LoVo cells $\left(5 \times 10^{7}\right)$ were injected subcutaneously in the right flanks of the nude mice. One week after tumor cell injection, equal amount of $r$ AAV-Luc-shRNA or rAAV-FHL2-shRNA was directly injected into the tumor at three different positions. 5 -FU ( $50 \mu \mathrm{g} / \mathrm{kg}$, once per two days, 7 times) or NS were endured by intraperitoneal injection at the same time. Photographs shown were taken on day 35 after rAAV injection. (B) The tumor size of mice with different treatments was calculated as follows: V=(4/3) R1 ${ }^{2} \mathrm{R} 2$, where $\mathrm{R} 1$ was radius 1 and $\mathrm{R} 2$ was radius 2 and $\mathrm{R} 1<\mathrm{R} 2$. Data are 42 -day tumor volumes for each 4 animals per group. ${ }^{*} \mathrm{P}<0.05$, rAAV-FHL2-shRNA vs $r \mathrm{AAV}$-LucshRNA. ${ }^{*} \mathrm{P}<0.05, r \mathrm{AAV}-\mathrm{Luc}$-shRNA + 5-FU vs rAAV-FHL2-shRNA + 5-FU. (C) Tumor size was measured weekly after tumor cell inoculation in each group. The arrow indicates the time when intratumoral and intravenous injections were performed.

These data suggest that rAAV-shRNA-FHL2-transduction with 5-FU treatment is sufficient to suppress tumorigenesis.

\section{Discussion}

Identification of the differences in the genetics of cancer cells with normal cells and exploration of cancer-associated genes are important for the development of targeted therapies in cancer treatment. Nude mice have been used extensively in cancer research because they do not reject allografts and often do not reject xenografts, making them an invaluable animal model to assess the therapeutic effect of novel molecules before human clinical trials. Here, we have demonstrated the anti- tumor efficacy of FHL2 inhibition by rAAV-shRNA in cells and nude mouse xenograft models through inducing cell cycle arrest and apoptosis, yielding that rAAV-shRNA-FHL2 might be a novel and potent therapeutic or 5-FU co-therapeutic agent for colon cancer.

As shown in our previous study, FHL2 was an oncogene in gastrointestinal cancers and inhibited cell differentiation and induced tumorigenesis (19), methods targeting FHL2 might be a promising strategy for the treatment of GI cancers. AAV is a non-enveloped, single-strand DNA virus, which belongs to the family Parvoviridae. AAV was used as our gene delivery vector because it offered several advantages over other delivery methods. First, it can infect a wide range 
of host cells irrespective of their cell cycle stages. Second, it mediates long-term gene expression. Third, when coupled to a strong promoter like the hybrid CMV enhancer/chicken $\beta$-actin (CAG) promoter used in this study, it is capable of delivering high levels of transgene expression in a wide variety of cell types. For these reasons, AAV carrying therapeutic genes like VEGF-Trap, AAV-hTERT-TRAIL and endostatin have been employed in the treatment of malignant glioblastoma, hepatic carcinoma and pancreatic cancer with varying degree of success.

Before in vivo study, the antitumor effect of rAAV-FHL2-shRNA was evaluated in vitro. Two key classes of regulatory molecules, cyclins and cyclin-dependent kinases (CDKs), determine a cell's progress through the cell cycle. Consistent with studies from other groups $(17,23)$, we found that rAAV-FHL2-shRNA inhibited cell cycle progression and caused significant G0/G1 arrest by inhibiting cyclin D1 and CDK6incoloncancercells(Fig.2A andB);rAAV-FHL2-shRNA induced G0/G1 arrest was also contributed to the inhibition of cell proliferation time- and serum-dependently (Fig. 2C and D). Next, rAAV-FHL2-shRNA induced apoptosis was revealed by western blot analysis and caspase activity assay (Fig. 3). In general, there are two well-characteristic pathways of apoptosis that are termed 'extrinsic pathway' and 'intrinsic pathway'. The former is triggered by the interaction between the membrane death receptors such as Fas, TNF-RI, DR3, DR4, DR5 and caspase 8 and their respective ligands. Regarding the intrinsic pathway, also known as the mitochondrial pathway, involves the increase of the mitochondrial permeability and the release of apoptogenic molecules such as cytochrome $c$ and Smac/DIABLO from mitochondria into cytosol, resulting in the activation of pro-caspase 9 and the downstream caspases. Activity of caspases 3, 8 and 9 were significantly higher in rAAV-FHL2-shRNA group, indicating that apoptosis induced by FHL2 inhibition was through both intrinsic and extrinsic pathway (31-33).

The effect of rAAV-FHL2-shRNA with or without chemotherapy 5-FU treatment on colon cancer was investigated in vitro and in vivo (Figs. 4 and 5) (29,34). Although few studies have shown the antitumor effect of FHL2 inhibition in different cancers, to the best of our knowledge there are no reports regarding therapeutic value of $\mathrm{rAAV}-\mathrm{FHL} 2$-shRNA in colon cancer. Inhibition of FHL2 by rAAV-FHL2-shRNA, as a long-term effective and specific targeting method, inhibited colon tumorigenesis and enhanced 5-FU-induced apoptosis in vitro. In vivo, it inhibited xenograft tumorigenesis and resulted in near eradication of established colon cancer xenograft when combined with 5-FU.

In conclusion, we have demonstrated that suppression of FHL2 by rAAV-FHL2-shRNA decreases colon cancer cell growth and cell cycle in vitro, and hinder tumor progression and outright regression in combination with 5-FU in vivo. Therefore, rAAV-FHL2-shRNA is potentially an important molecular target for the design of novel anti-colon cancer therapy.

\section{Acknowledgements}

This study was supported the National Natural Science Foundation of China (81172057 and 81272761), 'President Foundation of Nanfang Hospital, Southern Medical University'
(2012B009), a high-level topic matching funds of Nanfang Hospital (2010036 and G201227).

\section{References}

1. Samson T, Smyth N, Janetzky S, Wendler O, Müller JM, Schüle R, von der Mark H, von der Mark K and Wixler V: The LIM-only proteins FHL2 and FHL3 interact with alpha- and beta-subunits of the muscle alpha7betal integrin receptor. J Biol Chem 279: 28641-28652, 2004

2. Labalette C, Nouet Y, Sobczak-Thepot J, Armengol C, Levillayer F, Gendron MC, Renard CA, Regnault B, Chen J, Buendia MA and Wei Y: The LIM-only protein FHL2 regulates cyclin D1 expression and cell proliferation. J Biol Chem 283: 15201-15208, 2008.

3. Chan KK, Tsui SK, Lee SM, Luk SC, Liew CC, Fung KP, Waye MM and Lee CY: Molecular cloning and characterization of FHL2, a novel LIM domain protein preferentially expressed in human heart. Gene 210: 345-350, 1998.

4. Sanchez-Garcia I, Osada H, Forster A and Rabbitts TH: The cysteine-rich LIM domains inhibit DNA binding by the associated homeodomain in Isl-1. EMBO J 12: 4243-4250, 1993.

5. Bach I: The LIM domain: regulation by association. Mech Dev 91: 5-17, 2000.

6. Johannessen M, Moller S, Hansen T, Moens U and Van Ghelue M: The multifunctional roles of the four-and-a-half-LIM only protein FHL2. Cell Mol Life Sci 63: 268-284, 2006.

7. Morlon PA and Sassone-Corsi P: The LIM-only protein FHL2 is a serum inducible transcriptional coactivator of AP-1. Proc Natl Acad Sci USA 100: 3977-3982, 2003.

8. Fimia GM, Cesare DDe and Sassone-Corsi P: A family of LIM-only transcriptional coactivators: tissue-specific expression and selective activation of CREB and CREM. Mol Cell Biol 20: 8613-8622, 2000

9. Yan J, Zhu J, Zhong H, Lu Q, Huang C and Ye Q: BRCA1 interacts with FHL2 and enhances FHL2 transactivation function. FEBS Lett 553: 183-189, 2003.

10. Du X, Hublitz P, Günther T, Wilhelm D, Englert C and Schüle R: The LIM-only coactivator FHL2 modulates WT1 transcriptional activity during gonadal differentiation. Biochim Biophys Acta 1577: 93-101, 2002.

11. Stilo R, Leonardi A, Formisano L, Jeso B Di, Vito P and Liguoro D: TUCAN/CARDINAL and DRAL participate in a common pathway for modulation of NF-kappaB activation. FEBS Lett 521: 165-169, 2002.

12. Purcell NH, Darwis D, Bueno OF, Müller JM, Schüle R and Molkentin JD: Extracellular signal-regulated kinase 2 interacts with and is negatively regulated by the LIM-only protein FHL2 in cardiomyocytes. Mol Cell Biol 24: 1081-1095, 2004.

13. McLoughlin P, Ehler E, Carlile G, Licht JD and Schafer BW: The LIM only protein DRAL/FHL2 interacts with and is a corepressor for the promyelocytic leukemia zinc finger protein. J Biol Chem 277: 37045-37053, 2002.

14. Philippar U, Schratt G, Dieterich C, Müller JM, Galgóczy P, Engel FB, Keating MT, Gertler F, Schüle R, Vingron M and Nordheim A: The SRF target gene Fhl2 antagonizes RhoA/MAL-dependent activation of SRF. Mol Cell 16: 867-880, 2004

15. Yang Y, Hou H, Haller EM, Nicosia SV and Bai W: Suppression of FOXO1 activity by FHL2 through SIRT1-mediated deacetylation. EMBO J 24: 1021-1032, 2005.

16. Genini M, Schwalbe P, Scholl FA, Remppis A, Mattei MG and Schafer BW: Subtractive cloning and characterization of DRAL, a novel LIM-domain protein down-regulated in rhabdomyosarcoma. DNA Cell Biol 16: 433-442, 1997.

17. Martin BT, Kleiber K, Wixler V, Raab M, Zimmer B, Kaufmann $M$ and Strebhardt K: FHL2 regulates cell cycle-dependent and doxorubicininduced p21Cip1/Waf1 expression in breast cancer cells. Cell Cycle 6: 1779-1788, 2007.

18. Kinoshita M, Nakagawa T, Shimizu A and Katsuoka Y: Differently regulated androgen receptor transcriptional complex in prostate cancer compared with normal prostate. Int J Urol 12: 390-397, 2005.

19. Wang J, Yang Y, Xia HH, Gu Q, Lin MC, Jiang B, Peng Y, Li G, An X, Zhang Y, Zhuang Z, Zhang Z, Kung HF and Wong BC: Suppression of FHL2 expression induces cell differentiation and inhibits gastric and colon carcinogenesis. Gastroenterology 132: 1066-1076, 2007. 
20. Li M, Wang J, Ng SS, Chan CY, Chen AC, Xia HP, Yew DT, Wong BC, Chen Z, Kung HF and Lin MC: The four-and-ahalf-LIM protein 2 (FHL2) is overexpressed in gliomas and associated with oncogenic activities. Glia 56: 1328-1338, 2008.

21. Ding L, Wang Z, Yan J, Yang X, Liu A, Qiu W, Zhu J, Han J, Zhang H, Lin J, Cheng L, Qin X, Niu C, Yuan B, Wang X, Zhu C, Zhou Y, Li J, Song H, Huang C and Ye Q: Human four-and-a-half LIM family members suppress tumor cell growth through a TGFbeta-like signaling pathway. J Clin Invest 119: 349-361, 2009 .

22. Scholl FA, McLoughlin P, Ehler E, de Giovanni $\mathrm{C}$ and Schafer BW: DRAL is a p53-responsive gene whose four and a half LIM domain protein product induces apoptosis. J Cell Biol 151: 495-506, 2000.

23. Amann T, Egle Y, Bosserhoff AK and Hellerbrand C: FHL2 suppresses growth and differentiation of the colon cancer cell line HT-29. Oncol Rep 23: 1669-1674, 2010.

24. Zhang W, Jiang B, Guo Z, Sardet C, Zou B, Lam CS, Li J, He M, Lan HY, Pang R, Hung IF, Tan VP, Wang J and Wong BC: Four and a half LIM protein 2 (FHL2) promotes invasive potential and epithelial-mesenchymal transition in colon cancer. Carcinogenesis 31: 1220-1229, 2010.

25. Zhang W, Wang J, Zou B, Sardet C, Li J, Lam CS, Ng L, Pang R, Hung IF, Tan VP, Jiang B and Wong BC: Four and a half LIM protein 2 (FHL2) negatively regulates the transcription of E-cadherin through interaction with Snail1. Eur J Cancer 47: 121-130, 2011.

26. Takada M, Nakamura Y, Koizumi T, Toyama H, Kamigaki T, Suzuki Y, Takeyama Y and Kuroda Y: Suppression of human pancreatic carcinoma cell growth and invasion by epigallocatechin-3-gallate. Pancreas 25: 45-48, 2002.

27. Ewald JA, Desotelle JA, Church DR, Yang B, Huang W, Laurila TA and Jarrard DF: Androgen deprivation induces senescence characteristics in prostate cancer cells in vitro and in vivo. Prostatex 73: 337-345, 2012.
28. Kar S, Palit S, Ball WB and Das PK: Carnosic acid modulates $\mathrm{Akt} / \mathrm{IKK} / \mathrm{NF}-\mathrm{\kappa B}$ signaling by PP $2 \mathrm{~A}$ and induces intrinsic and extrinsic pathway mediated apoptosis in human prostate carcinoma PC-3 cells. Apoptosis 7: 35-47, 2012.

29. Chao TC and Greager JA: Experimental pulmonary sarcoma metastases in athymic nude mice. J Surg Oncol 65: 123-126, 1997.

30. Wendtner CM, Kofler DM, Theiss HD, Kurzeder C, Buhmann R, Schweighofer C, Perabo L, Danhauser-Riedl S, Baumert J, Hiddemann W, Hallek M and Büning H: Efficient gene transfer of CD40 ligand into primary B-CLL cells using recombinant adeno-associated virus (rAAV) vectors. Blood 100: 1655-1661, 2002.

31. Chatfield $\mathrm{K}$ and Eastman A: Inhibitors of protein phosphatases 1 and $2 \mathrm{~A}$ differentially prevent intrinsic and extrinsic apoptosis pathways. Biochem Biophys Res Commun 323: 1313-1320, 2004.

32. Mühlethaler-Mottet A, Bourloud KB, Auderset K, Joseph JM and Gross N: Drug-mediated sensitization to TRAIL-induced apoptosis in caspase-8-complemented neuroblastoma cells proceeds via activation of intrinsic and extrinsic pathways and caspase-dependent cleavage of XIAP, Bcl-xL and RIP. Oncogene 23: 5415-5425, 2004.

33. Obexer P, Geiger K, Ambros PF, Meister B and Ausserlechner MJ: FKHRL1-mediated expression of Noxa and Bim induces apoptosis via the mitochondria in neuroblastoma cells. Cell Death Differ 14: 534-547, 2007.

34. Guo XL, Li D, Hu F, Song JR, Zhang SS, Deng WJ, Sun K, Zhao QD, Xie XQ, Song YJ, Wu MC and Wei LX: Targeting autophagy potentiates chemotherapy-induced apoptosis and proliferation inhibition in hepatocarcinoma cells. Cancer Lett 320: 171-179, 2012. 